adenovirus were reduced in size and weight $(P<0.005)$ and contained fewer synoviocytes and mononuclear cells. The number of apoptotic cells in tissues treated with the FASLG adenovirus was increased 15-20-fold compared with controls.

Although adenoviral vectors are immunogenic-as evidenced by a small increase in the frequency of apoptotic cells after injection with the control adenovirus-and, therefore, not likely to be optimal for gene transfer in humans, this study highlights the potential of molecular surgery for the successful treatment of RA and paves the way for further refinement of the technique.

Rebecca Doherty

Original article Zhang $\mathrm{H}$ et al. (2005) Elimination of rheumatoid synovium in situ using a Fas ligand 'gene scalpel'. Arthritis Res Ther 7: R1235-R1243

\section{Manifestations of Churg-Strauss syndrome might vary according to ANCA status}

A prospective, multicenter study has suggested that patients with Churg-Strauss syndrome who test positive for antineutrophil cytoplasmic antibodies (ANCAs) might be phenotypically different to those who test negative for these antibodies. No previous large studies have evaluated the association of ANCA status with clinical characteristics and outcome of patients with this rare, small-vessel vasculitis.

A total of 112 patients with recently diagnosed Churg-Strauss syndrome from 63 clinical centers in France, Belgium, Latvia and the UK, were enrolled in a therapeutic trial of cyclophosphamide. At study entry (between December 1995 and December 2002), patients were assessed for the presence of ANCAs in serum and for clinical symptoms and manifestations of Churg-Strauss syndrome.

ANCAs were identified in 43 patients (38\%). When ANCA-positive patients were compared with ANCA-negative patients, they were more likely to have peripheral neuropathy (84\% versus $65 \%$ ) and renal manifestations (35\% versus $4 \%$ ), but were less likely to have fever (30\% versus $55 \%$ ) and cardiac manifestations (12\% versus $49 \%$ ).

The therapeutic trial is ongoing, and therefore no information is yet available about the outcome of treatment in the two groups of patients. The authors suggest that a better understanding of the pathogenetic mechanisms behind the differences in clinical manifestations might be useful in the development of future therapeutic strategies.

Rebecca Doherty

Original article Sablé-Fourtassou R et al. (2005)

Antineutrophil cytoplasmic antibodies and the Churg-

Strauss syndrome. Ann Intern Med 143: 632-638

\section{Serum antibodies and mortality rate in patients with rheumatoid arthritis}

Diagnosis of rheumatoid arthritis (RA) has historically depended upon clinical manifestations of the disease. Identification of factors that could predict severe disease and death is a high priority, as it would enable treatment to be initiated earlier. Presence and/or serum levels of rheumatoid factor (RF), antineutrophil cytoplasmic antibodies (ANCA) and the antibody against cyclic citrullinated peptide (anti-CCP) are all associated with disease severity; anti-CCP can be detected in serum even before the onset of clinical symptoms. Sihvonen et al. are the first to examine whether these factors are also associated with RA-related death.

Using baseline sera from 604 patients with RA who had participated in a 1988 Finnish study of renal and urinary tract comorbidity, the investigators tested for the presence of RF $(n=604)$, $\mathrm{RF}$ isotypes (IgA RF, IgG $R F$ and $\lg M R F$, $n=206)$, anti-CCP $(n=184)$ and ANCA $(n=200)$. A mortality cutoff date of 31 August 1999 was set, and government statistics revealed 160 patients (26\%) had died. Multivariate analysis showed that positive RF status $(P=0.011)$ and high levels of $\lg \mathrm{A}(P=0.003)$ and $\operatorname{lgM}(P=0.006)$ $\mathrm{RF}$ isotypes were predictors of death, but anti-CCP and ANCA levels were not.

These results indicate that patients with RA who have high immunologic activity are at an increased risk of death. As mean duration of $R A$ in these patients was $15 \pm 10$ years when the study began, studies measuring immunologic activity at diagnosis are recommended, in order to confirm the predictive value of these findings.

Katherine Sole

Original article Sihvonen S et al. (2005) The predictive value of rheumatoid factor isotypes, anti-cyclic citrullinated peptide antibodies, and antineutrophil cytoplasmic antibodies for mortality in patients with rheumatoid arthritis. J Rheumatol 32: 2089-2094 